Weiter hat es sich bei Verfahrenseinstellungen, die wegen des Zeugnisverweigerungsrechts der Geschädigten erfolgen müssen, als sinnvoll erwiesen, die Geschädigte und den Beschuldigten über die Möglichkeit einer Wiederaufnahme des Verfahrens zu informiert. Eine Einstellung gemäß $\mathbb{S} 153$ Absatz $1^{12}, 170$ Absatz $2^{13}$ StPO bewirkt keinen Strafklageverbrauch ${ }^{14}$, sodass bei Änderung der Beweislage, insbesondere wenn sich das Opfer zu einem späteren Zeitpunkt zur Aussage entschließt, das Verfahren wieder aufgenommen und auch angeklagt werden kann, solange noch keine Verjährung eingetreten ist. ${ }^{15}$ Dies wird auch tatsächlich in der Praxis so umgesetzt. Manchmal erst nach Jahren ist das Opfer an einem Punkt angelangt, an dem es verstanden hat, dass der Täter sich nicht mehr bessern wird. Und dass die Gewalt auch nicht noch viel schlimmer werden kann, als sie jetzt schon ist. Wenn sich das Opfer nun von dem gewalttätigen Partner trennt, folgt oft eine umfassende Aussage, auch über Vorfälle aus der Vergangenheit. Hier können dann die früher angelegten Verfahrensakten, die nach der Einstellung mangels Nachweises ins Archiv gelangten, wieder aufgenommen und zur Grundlage der Beweisführung gemacht werden. Die zu den damaligen Tatzeiten erhobenen Beweise sind jetzt wichtige Hilfsmittel: Die Geschädigte ist nach Jahren zwar aussagewillig, aber kann aufgrund der Vielzahl der ähnlichen Vorfälle oft Taten und Tatzeiten, Begehungsweisen und Umstände nicht mehr konkret und unterscheidbar benennen. Wenn ihr dann aber Vorhalte aus den alten Verfahrensakten gemacht werden können, kehrt die konkrete Erinnerung zurück, sodass die Taten anklagefähig beschrieben sind und abgeurteilt werden können.
In zahlreichen Sonderdezernaten bundesweit wird täglich dieselbe Erfahrung gemacht: Mit besonderem Hintergrundwissen, spezieller Ermittlungstaktik und vorausschauender Vorgehensweise lassen sich die Herausforderungen, vor die uns die Fälle häuslicher Gewalt stellen, meistern. Der Arbeit in den allgemeinen Abteilungen der Staatsanwaltschaften ist dagegen das zur Verfolgung häuslicher Gewalt notwendige Durchsetzen der Strafverfolgung gegen den Willen des Opfers fremd. Dort ist man eher daran gewöhnt, unrealistische Erwartungen von Geschädigten an eine möglichst hohe Bestrafung zurückzuweisen. Auch der Blick dafür, dass zunächst eher harmlos erscheinende Sachverhalte unter Umständen tiefe Abgründe und Hochrisikofälle verbergen können, entsteht erst während der intensiven Beschäftigung mit Fällen häuslicher Gewalt.

Unter Menschenrechtsgesichtspunkten, aber auch schon aus wirtschaftlichen Erwägungen heraus, ${ }^{16}$ kommt ein Staat nicht umhin, effektive Maßnahmen zur Bekämpfung von häuslicher Gewalt zu ergreifen. Dabei stellt eine konsequente und effektive Strafverfolgung einen wichtigen Baustein dar. Sie ist ohne Sonderdezernate bei den Staatsanwaltschaften nicht zu leisten.

12 Einstellung wegen geringer Schuld.

13 Einstellung mangels ausreichenden Nachweises.

14 Meyer-Goßner, Lutz, StPO, 53. Aufl. 2010, § 153 StPO Rn. 37, § 170 Rn. 9.

15 Vorsätzliche Körperverletzung verjährt erst nach fünf, gefährliche Körperverletzung nach zehn, Vergewaltigung nach 20 Jahren.

16 Häusliche Gewalt ist ein erheblicher Kostenfaktor, vgl. beispielsweise Schätzung des, National Center for Injury Prevention and Control' für die USA auf 5,8 Mrd. US-Dollar jährlich, <www.cdc.gov/ violenceprevention/pdf/IPVBook-a.pdf> (Zugriff: 16.1.2013).

\title{
Qualifizierung forensischer Sachverständiger durch die Psychotherapeutenkammern
}

\section{Marion Schwarz}

Diplom-Psychologin, Kinder- und Jugendlichenpsychotherapeutin, Psychologische Psychotherapeutin, Sachverständige für Familienrecht, Bad Schwalbach

Die Heilberufegesetze der Bundesländer sehen für die Kammern auch die Aufgabe vor, Sachverständigenlisten zu führen. Daher haben die Landespsychotherapeutenkammern vor fünf Jahren eine Arbeitsgruppe gegründet, die Kriterien für die Anerkennung und Fortbildung von forensischen Sachverständigen aus dem Mitgliederbereich der Psychotherapeut/inn/en ${ }^{1}$ entwickeln sollte. Diese AG erarbeitete ein Modell für die qualifizierte Fortbildung/Weiterbildung von Sachverständigen sowie Übergangsbestimmungen für die Anerkennung bereits tätiger Sachverständiger, jeweils bezogen auf ein Rechtsgebiet.

Die einzelnen Landeskammern sind nunmehr dabei, diese Musterrichtlinie durch jeweils eigene Fort- oder Weiterbildungsordnungen umzusetzen. ${ }^{2}$

\section{Modulare Fort-/Weiterbildung}

Die Fortbildung ist modular aufgebaut. Zunächst sollen über ein Grundlagenmodul allgemeine Rechtsgrundlagen und die Aufgaben als Sachverständige vermittelt werden, sowie spezifische psychologische Kenntnisse.

Darauf aufbauend werden verschiedene Spezialisierungsmodule angeboten:

- zum Strafrecht und Strafvollstreckungsrecht,

- zur Glaubhaftigkeit der Zeugenaussage,

vur Prognosebeurteilung,

- zum Familienrecht,

- zum Sozialrecht.

1 Psychologische Psychotherapeut/inn/en und Kinder- und Jugendlichenpsychotherapeut/inn/en.

2 Psychotherapeutenkammer Hessen-LPPKJP, Psychotherapeutenkammer Rheinland-Pfalz, Ostdeutsche PsychotherapeutenkammerOPK, Psychotherapeutenkammer NRW, Psychotherapeutenkammer Niedersachsen, Psychotherapeutenkammer Bremen, Psychotherapeutenkammer Bayern, Psychotherapeutenkammer Saarland. 
Daran anschließend sollen dann in der Regel fünf Gutachten unter Supervision angefertigt werden.

Mit dem abgeschlossenen Spezialisierungsmodul hat man dann die Qualifikation und Anerkennung als forensische/r Sachverständige/r für das jeweilige Rechtsgebiet erworben. Dies ist ein wesentlicher Unterschied zu den Rechtspsycholog/inn/en, die vom BDP/Sektion Rechtspsychologie ausgebildet werden.

\section{Bedeutung der Approbation für Sachverständigentätigkeit}

Die von den Kammern akkreditierten Sachverständigen sind alle approbierte Psychotherapeut/inn/en. Die psychotherapeutische Ausbildung erfolgt postgradual, also nach einem Studium. Für die Ausbildung zur/zum Psychologischen Psychotherapeutin/Psychotherapeuten ist das Psychologie-Studium Voraussetzung, für die Kinder- und Jugendlichenpsychotherapeut/ inn/en ebenso oder auch ein Studium der Pädagogik/Sozialpädagogik. Die Ausbildung dauert drei Jahre als Vollzeitausbildung und mindestens fünf Jahre berufsbegleitend. Sie wird mit einer Staatsprüfung abgeschlossen (Approbation).

Diese zusätzliche berufliche Qualifikation ist im Vergleich zur Rechtspsychologin/zum Rechtspsychologen (BDP), die/ der diese Fortbildung als Diplom-Psychologin oder DiplomPsychologe erwerben kann, ein weiterer Qualifikationsunterschied, der gerade auch angesichts der spezifischen Fragestellungen im Bereich der Sachverständigentätigkeit von Bedeutung sein dürfte.

Die Relevanz von psychischen Erkrankungen ist nicht nur im Maßregelvollzug gegeben, sondern zunehmend auch bei der Beurteilung von Straftäter/inne/n, sowie bei der Erarbeitung von Prognosen. Durch die psychotherapeutische Ausbildung ist die nötige qualifizierte klinische Fachkunde vorhanden, um diese spezifischen Fragestellungen zu untersuchen und eine Diagnose stellen zu können.

Auch in der Beurteilung von Glaubhaftigkeit von Zeugenaussagen wird es von Bedeutung sein, ob die- oder derjenige psychisch gesund ist oder ob ggf. eine Erkrankung gemäß ICD- $10^{3}$ vorliegt.

Gerade auch im Bereich des Familienrechts kann festgestellt werden, dass bei hochkomplexen Fällen, die sich gerade nicht durch vorherige Mediation oder Ähnliches lösen lassen, ein deutlicher Anteil von Eltern beteiligt ist, die eine psychische Störung aufweisen. Zudem erscheint es in diesem Rechts- und Fachgebiet von Bedeutung, dass die/der Sachverständige ausgewiesene Kenntnisse in der Diagnostik und Behandlung von Kindern und Jugendlichen nachweisen kann. Diese Kriterien erfüllen in besonderem Maße die approbierten Kinder- und Jugendlichenpsychotherapeut/inn/en. Kinder und Jugendliche sind durch die Auseinandersetzungen zwischen ihren Eltern in Fragen der elterlichen Sorge, des Umgangs etc. oftmals selbst hoch belastet. Daraus resultierende Folgestörungen können sehr unterschiedlich ausfallen und die Haltung des Kindes oder der/des Jugendlichen hinsichtlich der gerichtlichen Fragestellung stark beeinflussen. Zudem sind Kinder- und Jugendlichenpsychotherapeut/inn/en aufgrund ihrer Berufskenntnisse mit der Arbeit hoch zerstrittener schwieriger Elternpaare vertraut. Psychologische Psychotherapeut/inn/en als Sachverständige im Familienrecht sollten demnach besondere Kenntnisse in der Behandlung und psychotherapeutischen Arbeit mit Kindern, Jugendlichen und Familien haben.

Auch in Fragen des Sozialrechts, beispielsweise der Berentung aufgrund einer psychischen Erkrankung oder aufgrund eines Unfalls mit folgender schwerer Traumatisierung, spielt die klinische und psychotherapeutische Kenntnis einer/eines Sachverständigen eine große Rolle. Sehr klar erscheint die Notwendigkeit psychotherapeutischer Kompetenz hinsichtlich der Beurteilung bei Transsexuellen durch Sachverständige.

\section{Qualitätssicherung durch die Kammer}

Um eine nachhaltige qualifizierte Sachverständigentätigkeit zu gewährleisten, haben die Kammern sich darauf verständigt, die Anerkennung von Sachverständigen zu befristen. In einem Zeitraum von fünf Jahren muss eine entsprechende gutachterliche Tätigkeit nachgewiesen werden sowie fachspezifische Fortbildung. Dies soll dazu beitragen, die Güte der Qualität der Sachverständigengutachten erhöhen.

\section{Übergangsvorschriften}

Auch Sachverständige, die bereits seit längerer Zeit tätig sind, können auf die durch die Kammer geführte Sachverständigenliste aufgenommen werden. Hierzu ist es erforderlich, eine festgesetzte Anzahl von Gutachten, die nicht älter als drei Jahre sein sollen, einzureichen. Ein Prüfungsausschuss überprüft nach festgelegten Kriterien die Qualität der eingereichten Unterlagen. Entsprechen die vorgelegten Gutachten den Qualitätsanforderungen, empfiehlt der Ausschuss dem Kammervorstand die Anerkennung als Sachverständige/r für das entsprechende Rechtsgebiet durch die Psychotherapeutenkammer.

\section{Literaturhinweise}

- H. Diling, W. Mombour, M.H. Schmidt, E. Schulte-Markwort (Hrsg.), 2011, Internationale Klassifikation psychischer Störungen, ICD-10, Kapitel V (F), Verlag Hans Huber.

- A. Boetticher, H.-L. Kröger, R. Müller-Isberner, K. Böhm, R. Müller-Metz, 2006, Mindestanforderungen für Prognosegutachten, Neue Zeitschrift für Strafrecht, Heft 10.

- A. Boetticher, H.A.G. Bosinski, N. Nedopil, H. Saß, 2007, Mindestanforderungen für Schuldfähigkeitsgutachten, Forensische Psychiatrische Psychologische Kriminologie 1.

3 ICD 10: Internationale statistische Klassifikation der Krankheiten und verwandter Gesundheitsprobleme; ICD-10 Kapitel V (F): Internationale Klassifikation psychischer Störungen. 